\title{
Study of Personal and Social Adjustment Ability of the Disabled Pupils
}

\author{
Natalia Vladimirovna Ryabova ${ }^{1} \&$ Tatyana Aleksandrovna Parfyonova ${ }^{1}$ \\ ${ }^{1}$ Mordovian State Pedagogical Institute named after M.E. Evseviev, Mordovia, Russia \\ Correspondence: Natalia Vladimirovna Ryabova, Mordovian State Pedagogical Institute named after M.E. \\ Evseviev, 430007 Mordovia, Russia.
}

Received: November 292014 Accepted: January 62015 Online Published: April 28, 2015

doi:10.5539/ies.v8n5p213

URL: http://dx.doi.org/10.5539/ies.v8n5p213

\begin{abstract}
The article presents the results of experimental work aimed at studying personal and social adjustment of a child with disabilities. Analysis of domestic and foreign psychological and educational literature allowed us to determine the key concept of the study, namely "social and personal orientation", as a trait of a human's personality, providing the ability to perceive the social environment, be involved into this environment and to master a variety of activities. The availability of this trait helps a child with disabilities to adapt into present-day social medium, thus solving the problem of his socialization. The paper describes the structure of readiness for personal and social adjustment, represented by personal, cognitive and activity components. A methodology of summative assessment, aimed at exploring the level of maturity of each of these components, as well as their integrity in the overall readiness structure, was designed and developed with due consideration of these components. Criteria and evaluation indicators, as well as special techniques (survey, interview and practice-oriented task) were selected to identify the level of maturity of each readiness component (high, medium, low and immature). The article presents the results of summative assessment, conducted with the help of the developed technique. Generalization of the obtained results indicates that the majority of children with disabilities, who were tested in the experiment, have low level of personal, cognitive and activity readiness components for personal and social adjustment. This suggests that pupils with disabilities show interest in contemporary social and welfare changes in a society, but do not seek to cognize the social medium on their own; moreover, most of them are immature in terms of knowledge of objects and phenomena of social realm; they have no need to expand the sources of information that would allow them to explore the world and various types of social and personal activities. In this regard, there appears a necessity to develop and implement targeted correction pedagogic work, providing formation of such a socialization mechanism, as personal and social adjustment that must be implemented under conditions of educational institutions, where today are taught many children with disabilities.
\end{abstract}

Keywords: socialization, personal and social adjustment, readiness, child with disability, methodology and results of summative assessment

\section{Introduction}

The problem of training, education and socialization of children with disabilities has become one of the priorities of the Russian state and society, since the number of children from this category does not decrease. According to the Department of State Policy in the field of children's rights protection of the Ministry of Education and Science of the Russian Federation, in the Russian educational institutions there are 467,176 children with disabilities, including 210,194 children, studying in 1,676 special (correctional) institutions; 110,192 children, studying in special classes at secondary schools; and 146,790 children, studying in general education classes at secondary schools. At the same time, changes in the socio-cultural situation in contemporary international space and Russian society have led to the fact that the state cannot ignore the problems of disabled persons. Today's public consciousness starts to realize that the health defect is not a stalemate, but the state, which can be controlled and whose possible negative effects can be significantly reduced, if necessary measures are taken, including those of psycho-pedagogical nature.

Today it is evident that the problem of care for disabled people is not so much in taking care of them all the time, but in creating conditions for their personal self-realization, socially significant activities or work. In a legal document, such as "National Educational Initiative 'Our New School", approved by the President of the Russian Federation on February 4th, 2010, \#271, it is stated that "The new school is a school for everyone. Any school 
will provide the successful socialization of children with disabilities, handicapped children and children without parental care, who are in hardship" (Executive Order of the President of the Russian Federation "National Educational Initiative "Our New School”, 2010).

Analysis of native literature (Malofeev, 2010; Nazarov, 2000, Shipitsyna, 2003, Shmatko, 2010, etc.) and foreign publications (Al-Rossan, 2012; Norwich, 2008; Humphrey, 2008; Lynch, 2009; Rogers, 2013; Abbott, 2007; Allan, 2006; Mintz, 2007; Thomazet, 2009; Mitchell, 2004; Lynch \& Irvine, 2009; and etc.), dealing with psychological and educational issues, allows us to emphasize the scientific and methodological bases of training and education of noted children in a "new" school. This implies exclusion of any discrimination against children regardless of their social status, physical, emotional and intellectual development; creation of special conditions for children on the basis of psycho-pedagogical techniques, focused on their needs; providing opportunities for all children to participate equally in the academic and social life; providing conditions for successful life sustaining activity and the socialization of children in today's society.

In our study, socialization is considered as the process of entering a person into a social environment, assimilation of social life experience, culture and social relationships (Andreeva, 2009). One of the mechanisms of its implementation is a personal and social adjustment. In our study a concept of "personal and social adjustment" is considered by comparing a number of concepts that form the following contexts: first, the "socialization", "social adjustment", "adjustment", "personal and social adjustment", where social and personal orientation serves one of the socialization mechanisms; second, the "activity", "orientating basis of activity", "social and personal activity", where social and personal orientation is indicative basis of social activities; third, "orientation", "social and personal orientation", "a system of social and personal orientation", where social and personal orientation appears as a means, providing the disabled pupil an orientation in the social environment and the acquisition of social activities with the help of specially developed content and pedagogical conditions, created for its implementation (Ryabova, 2007). In a generalized sense the studied concept is defined as “... an integrated hierarchical system of social needs and motives, which, on the one hand, defines the structure of values and subjective meanings, the specificity of relations with the world and to the world, the direction of the main lines of conduct; while on the other hand it is a product of the process and the result of socialization, which guarantees formation of readiness for social and personal orientation as the human personality trait that provides an opportunity to perceive the social environment, be involved into this environment and to master a variety of activities". The formation of this personality trait is possible through the development of readiness for social and personal orientation, which we regard as an forceful (active) condition of a personality, attitude towards a certain behavior, ability to mobilize forces in order to reach performance target, to actualize the necessary knowledge, as well as maturity of skills (Parfyonova, 2013).

The readiness structure is represented by three components: personal component (includes sustained outlook, as well as social and moral beliefs; flexibility in daily life situations; independence in searching and uptaking of social information, initiative and other personal qualities, providing him success in the life sustaining activity); cognitive component (includes knowledge of objects and phenomena of the immediate surround, knowledge about the people that make up the inner circle, social roles, including those related to occupational choice, and other knowledge providing familiarization with the society and the mastery of a variety of activities); and activity component (which includes general intellectual, labor and social skills, related to daily life, allowing one to acquire personal and social adjustment) (Ryabova \& Parfyonova, 2011).

We show the development of methodology and research results of the social and personal orientation of a disabled child in the course of summative assessment, conducted in 2010-2011 academic year in the Municipal Budgetary General Educational Institution Secondary Schools (MBGEI SS) \#37 and \#8 in Ulyanovsk city of the Ulyanovsk Region, the Russian Federation.

\section{Methodology}

The purpose of summative assessment was to investigate and identify the level of readiness of younger pupils with mental retardation (MR) towards social and personal orientation. Note that the category of children with mental retardation refers to one of the largest categories of disabled children.

The study of readiness was carried out taking into account the selected parameters, namely: firstly, the maturity of readiness components (personal, cognitive and activity), and secondly, the structural integrity of readiness. Let describe the criteria for determining the first parameter.

To assess the maturity of personal component we have defined criteria, which are represented by value and social orientations, motivational targeting, as well as health condition and personality traits. Personal readiness maturity indicators are as follows: sustained outlook; social and moral beliefs; flexibility in any social and daily 
life situation; ability to assimilate new information; independence; need to bring the undertaking to its logical conclusion; initiative; social responsibility, and others. Let consider examples of the tasks: "You had a quarrel with a friend. What would you do? "(value orientation of communication), "The teacher asked you to prepare a presentation, but on this day you planned to devote a lot of time doing homework in all subjects. What would you do?" (value orientation to knowledge), "What would you do if your friend spoiled a thing, belonging to teacher, and hided it?" (social orientation), and others.

To assess the maturity of cognitive readiness component, we have defined criteria, which are represented by the knowledge about the objects and phenomena of social environment, knowledge about the interaction with the outside world, etc. Indicators of cognitive readiness maturity include the completeness, depth, consistency, and awareness of acquired knowledge. The study was carried out individually, using the interview-based technique. For example, knowledge about the objects and phenomena of the social medium were studied by questions, such as "What belongs to nature?", "What is created by human?" etc., while the knowledge about the interaction between objects and phenomena in the surrounding world were checked through the questions, such as: "What kinds of transport do you know?", "What continents do you know?", etc.

To assess the status of activity readiness component, we have defined criteria, which were represented by matured skills (general intellectual skills, general labor skills, personal and social adjustment skills, and communicative skills), while indicators were differentiated as follows: the ability to organize own learning activity; ability to carry out an indicative framework for action (understanding the essence of the educational material under study: analysis, synthesis, abstraction, specification, induction, deduction, classification, generalization; reasoning, formulation of conclusions and inferences, solving problems and tasks, etc.); ability to plan learning activity (recognition of the learning task, goal setting, choice of a rational and optimal ways to achieve the objectives, definition of the sequence and duration of activity stages, construction of activity algorithm, etc.); the ability to schedule an intercommunication. The study of the activity component was carried out individually based on pupils' practice-oriented activity, nonscheduled in terms of time, in accordance with the specific task. For example, "Circle and engrave figure (circle) on a piece of paper", "Draw a square with side of $5 \mathrm{~cm}$ ", "Lay the table to breakfast", "Iron a shirt", etc.

Having all the indicators we can talk about the full manifestation of the criterion; manifestation of the main indicators makes it possible to assess the appropriate criterion manifestation level; when determining just one indicator (or complete lack thereof) we can assume that given criterion is lacking (Makarova, 2000).

We have identified four levels of readiness for social and personal orientation of disabled pupils, namely, high, medium, low and immature level. At a high level of readiness, all the qualities that make up readiness of disabled pupils are matured; pupils are ready to work on social and personal orientation; they understand its essence and have social and daily life knowledge, enabling them to carry out various kinds of social and personal activities. At an average level of readiness, pupils with disabilities have positive attitude towards the activity on social and personal orientation, they exhibit value orientation, social and moral beliefs; children have social and living knowledge, but find some difficulties in knowledge transfer; they are able to perform various kinds of social and personal activities, but have difficulties in constructing an algorithm on execution sequence of activities and operations. At a low level of readiness, pupils with disabilities have poorly formed all the quality groups that make up readiness; children are not oriented in the statement of the task, have some social and living knowledge, but do not know how to apply them in the social and personal activities. At immature level of readiness, pupils with disabilities not strive to meet the social and personal activities; they have social and daily life knowledge, required to carry out certain activities, related to the satisfaction of everyday and physiological needs; they perform activities, aimed at solving social problems, very poorly; children find difficulties in communication processes, do not master source of information, necessary to perform assigned tasks.

Next we describe the maturity of second parameter, which defines readiness to social and personal orientation, namely the integrity of readiness structure. Integrity of readiness to social and personal orientation among the pupils with disabilities is defined by the presence of each component (personal, cognitive and activity) and their coherence in the readiness structure. Integrity indicators include: 1) the level of maturity of each readiness component; 2) the presence of the major components in the readiness structure; 3) the relationship between the components of readiness structure. When revealing these indicators, we use the tier characteristic of maturity of the major readiness components.

Given these levels, the structural integrity of readiness for social and personal orientation can be represented as follows: 1) all of the readiness components are at a high level; 2) two readiness components are at a high level, while one component is at a middle level; 3 ) two readiness components are at a high level, while one component 
is at a low level; 4) one readiness component is at a high level, the second one is at the middle level, while the third one is at a low level; 5) one readiness component is at a high level, while the other two are at a middle level; 6) all of the readiness components are at a middle level; 7) two readiness components are at an average level, while one is at a low level; 8) one readiness component is at an average level, while the other two are at a low level; 9) all readiness components are at a low level; 10) at least one readiness component is immature, i.e. lacking at all (Table 1).

Table 1. Levels of structural integrity of readiness for social and personal orientation of pupils with disabilities

\begin{tabular}{ll}
\hline Levels & Level indicators \\
\hline 1 & All readiness components are at a high level. \\
2 & Two readiness components are at a high level, while one component is at a middle level. \\
3 & Two readiness components are at a high level, while one component is at a low level. \\
4 & $\begin{array}{l}\text { One readiness component is at a high level, the second one is at the middle level, while the third one is } \\
\text { at a low level. }\end{array}$ \\
5 & One readiness component is at a high level, while the other two are at a middle level. \\
6 & All readiness components are at a middle level. \\
7 & Two readiness components are at an average level, while one is at a low level. \\
9 & One readiness component is at an average level, while the other two are at a low level. \\
10 & All readiness components are at a low level. \\
\hline
\end{tabular}

Thus, taking into account the developed research technique to assess the maturity of the readiness components for social and personal orientation and their integrity in the readiness structure, we have carried out study, based on interview of forty $3^{\text {rd }}$ grade pupils with a diagnosis of mental retardation. Pupils were studying at Ulyanovsk secondary schools \#37 and \#8 in the Ulyanovsk Region of the Russian Federation. Let discuss the results of conducted summative assessment.

\section{Results}

The results of the research on personal readiness component of pupils with disabilities led to the following conclusions. First, all the surveyed pupils were differentiated into four groups (with high, medium, low and immature levels of personal readiness to social and personal orientation); second, the reviewed pupils showed certain qualitative differences in terms of maturity of personal readiness component.

The first group consists of the most preserving pupils, who have successfully coped with the solution to the problem situation. They have demonstrated the presence of both value orientation (interest in the activity, understanding and awareness of the need for activity, the desire for knowledge of reality), and social orientation (orientation in objects and phenomena of reality, consideration of the interests and needs of other people, commitment to mastering the cultural behavior, as well as social norms and rules). The results also showed the maturity of moral convictions (readiness to work, commitment to the assimilation of various kinds of social and personal activities, participation in useful activities, manifestation of the favor for labor, as well as initiative and creativity) and qualities, allowing the pupil to study the social and daily life reality (activity, diligence, conscientiousness, responsibility, positive attitude to work and working people).

The second group consists of relatively preserving pupils, who have partly coped with the task. They were interested in the activities, understood and realized the need for activity; oriented in objects and phenomena of reality, master cultural behavior, social norms and regulations; they expressed the need and willingness to work, striving for the assimilation of various kinds of social and personal activities, lined up algorithm of working operations, showed an orientation on the activity process and results; showed diligence, conscientiousness, responsibility; though pupils did not always seek for cognition of social realm, did not take into account the interests and needs of other people, they were involved in useful activities, though did not show independence and initiative, did not set goals to achieve a particular result.

The third group consists of pupils, who have experienced significant difficulties in development, as well as the 
availability of knowledge and skills that did not allow these children to cope successfully with the tasks. The kids had shaky attitude with regard to values, were not able to evaluate the outside world, did not have the ideas of what is important and worthwhile for a human; weakly expressed their interest in activities, did not understand and realize the need for it, did not not aim to achieve a particular result, did not know the algorithm of particular working operations, showed a weak orientation towards the process and results of operations; tested children did not interact with each other in various situations, showed lack of initiative, did not take responsibility, did not evaluate the response of others, had little idea about the social responsibilities; exhibited negative attitude characterizing the personality traits.

The fourth group consists of pupils, who have no interest in any activity, no need to communicate with others, no desire for knowledge of the surrounding society. They do not master social norms and rules, cultural behavior, do not seek to master the social and personal activities, and do not take the initiative and creativity.

Results of the personal readiness component maturity are shown in Figure 1.

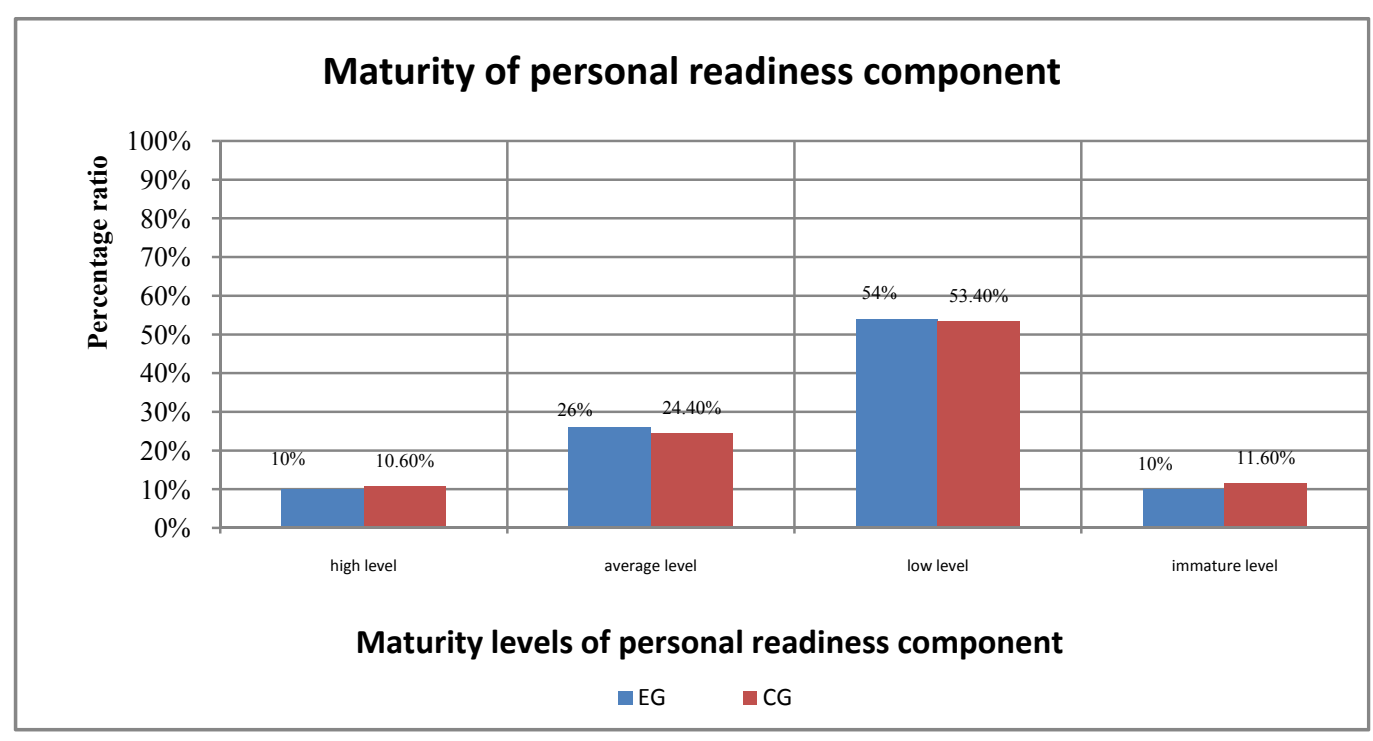

Figure 1. Maturity of personal readiness component

Note that the results of the study showed some qualitative differences in the maturity of the personal component. For example, pupils with disabilities have insufficient understanding of the value orientations, do not always show the ability to relate them to the real life situations; rarely evaluate both their own behavior and the behavior of other people; they do not have sustainable attitude towards moral values; children are poorly informed on issues related to socially significant personality traits; have some understanding of the social behavior patterns, partly orient in the communication situations, though often are passive; their personality traits are underdeveloped that makes it impossible to successfully interact with other people. Thus, the cumulative result obtained in the study suggests that personal component of readiness for social and personal orientation of the majority of pupils with disabilities is low.

Analysis of the research conducted to study the cognitive component of readiness of disabled children led to the following conclusions: first, all surveyed pupils are differentiated into four groups (with high, medium, low and immature levels of cognitive readiness for social and personal orientation); second, the tested pupils showed some qualitative differences in maturity of the cognitive component of readiness.

At a high level of cognitive readiness for social and personal orientation, pupils with disabilities have social and everyday living knowledge needed to carry out various kinds of social and personal activities. This level corresponds to the answers, in which children summarized the existing knowledge on the issue, made the choice of an adequate answer to the question posed; gave answers that included correct, complete, accurate, consistent presentation of information; and considered the objects holistically. At an average level of cognitive readiness, pupils with disabilities have social and daily living knowledge, though find it difficult to transfer this knowledge; children understand statement of a problem or a task, but their responses lack complete information, including 
only basic knowledge, not always adequately revealing the essence of the problem. At a low level of cognitive readiness, pupils with disabilities are not oriented in the statement of the task, have some of the social and daily life knowledge, but do not know how this knowledge is used in social and everyday activities. Children do not establish links between objects; they are not able to update the existing knowledge on the problem, so often give inadequate answers. At immature cognitive readiness, pupils with disabilities have extremely limited social and daily life knowledge, necessary for personal social and everyday life needs, though they lack substantive knowledge, necessary for productive social and personal orientation and mastery of the main types of social and personal activities.

Results of the personal readiness component maturity are shown in Figure 2.

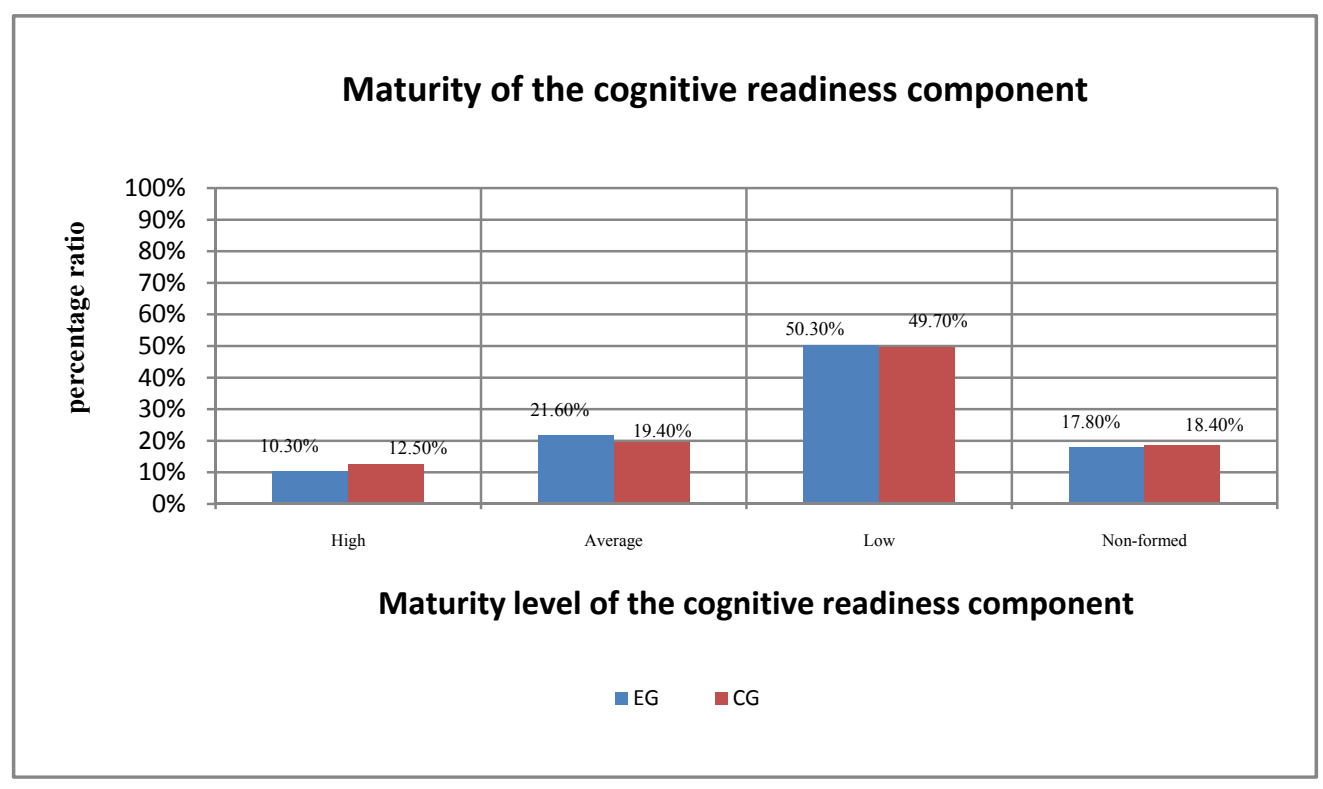

Figure 2. Maturity of the cognitive readiness component

Note that the results of the study showed some qualitative differences in the maturity of the cognitive component. Thus, children coped most successfully with issues relating to knowledge of the objects and phenomena of the social media, they had a fairly accurate perception of the outside world, knew the main features of the surrounding objects and phenomena, their properties and qualities; tested children showed good results in legal and socio-cultural issues that was manifested in their awareness of the social behavior patterns in the community, behavior rules in specific communication situations; pupils fairly well coped with the problems of social character, where they showed awareness of issues, related to socially important activities, as well as objects and materials, necessary for their implementation; tested pupils experienced considerable difficulties answering the questions concerning knowledge about the interaction of objects and phenomena in the surrounding world; children ran against problems when listing, comparing, and analyzing objects and phenomena of the outer world, found it difficult to select generalizing concepts and classify the concerned objects. Thus, the cumulative result obtained in the study suggests that cognitive component of readiness for social and personal orientation of the majority of pupils with disabilities is at a low level.

Results of the study, conducted on activity component of the disabled pupils' readiness, made it possible to make the following conclusions: first, all the surveyed pupils were differentiated into four groups (in terms of high, medium, low and immature levels of readiness for social and personal orientation); second, the tested pupils showed some qualitative differences in maturity of the activity component of readiness.

At a high level of the activity component, pupils with disabilities are focused on job in the social sphere, understand its essence, have social and everyday life knowledge, allowing them to carry out various kinds of social and personal activities. This category of children analyzed the problem, highlighting the main concept, could choose a rational way to solve it; determined the sequence of work stages, strictly adhering to them in the course of operations; were able to develop activity algorithm, adequately performing all manipulations and 
bringing the work to its logical end; if necessary, explained their actions; were able to transfer knowledge into practice, while using equipment and supplies for its intended purposes; carried out the work independently. At the average level of the activity component, disabled pupils have a positive attitude towards the activity on social and personal orientation, are able to perform various kinds of social and personal activities, though have difficulties in the algorithm constructing process. When performing tasks, tested children analyzed the problem posed, emphasizing the problem core, though not always carried out rational choice and the best ways to achieve results; made slight mistakes in the sequence of individual operations that did not affect the final result of the work; were able to use knowledge in practice; though working practices performed with some mistakes. At a low level of the activity component, disabled children demonstrated ability to carry out main types of social and personal activities; pupils did not comply with the consistency in their activities and made significant mistakes in individual operations; they did not support their work by the necessary knowledge, while practicing trials and conjectures. At the immature level of the activity component, pupils with disabilities were able to carry out various kinds of social and personal activities, necessary to ensure their own life sustaining activity, though could not transfer acquired skills into practical activities towards social and personal orientation.

Results of the cognitive readiness component maturity are shown in Figure 3.

\section{Maturity of the activity readiness component}

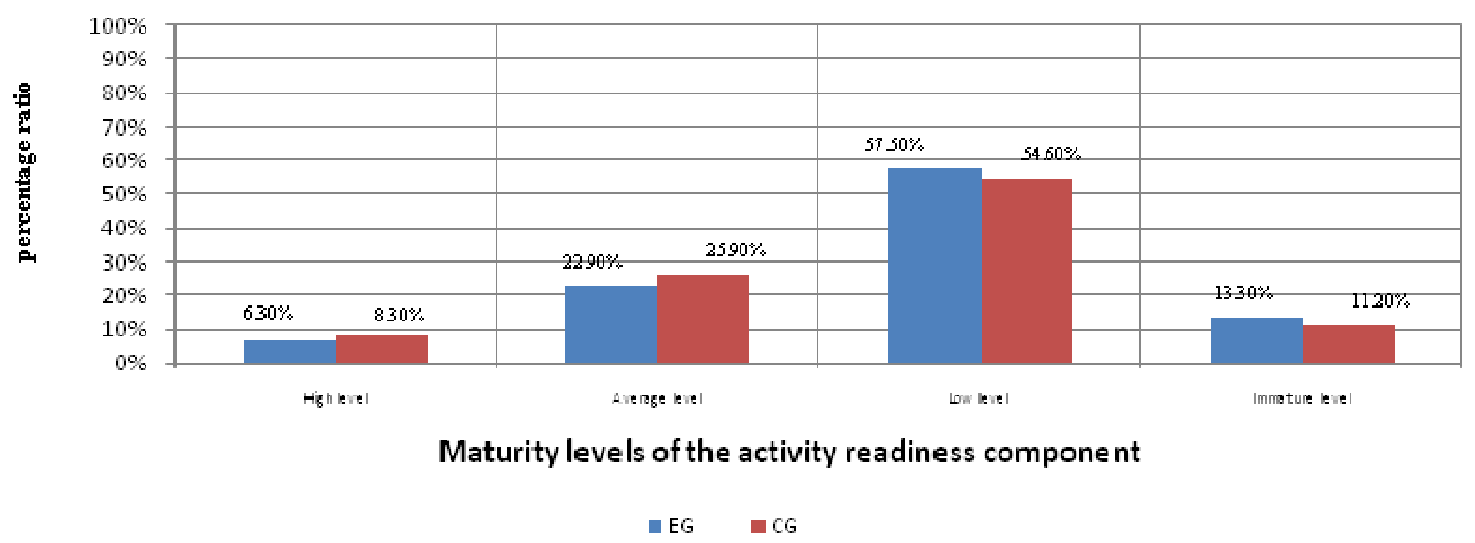

Figure 3. Maturity of the activity readiness component

Note that the results of the study showed some qualitative differences in the maturity of the activity component. Thus, children coped most successfully with the tasks relating to general intellectual skills (EG-31.6\%, CG-36.7\%), general occupational skills (EG-31.7\%, CG-36.6\%) and communication skills (EG-31.7\%, KG-36.7\%) showing almost identical results; considerable difficulties in tested children were caused by the tasks, in which pupils had to show the social and personal skills (EG-21.6\%, CG-26.7\%). In general, pupils with disabilities are able to carry out social and personal activities, though they have poorly developed practical skills, necessary to realize these activities (for example, pupils did not comply with the sequence in the course of activities, used to make significant mistakes in individual operations, this work was not supported by the necessary knowledge, because was based on the trials and conjectures); they are knowledgeable in matters, relating to training activities, though cannot fully apply them; have some idea of communicative interactions, are partially oriented in the communication situation, but often encounter difficulties in the new (unknown) situation. Thus, the cumulative result obtained in the study, suggests that activity component of readiness for social and personal orientation of the majority of pupils with disabilities is at a low level.

While giving a qualitative characteristic to the social and personal orientation readiness of disabled pupils, let dwell on its integrity. In the readiness structure, there is a different hierarchy of personal, cognitive and activity components, which may be matured at a high, medium and low levels in a variety of presentation options, as well as represent a situation, where one of the components is immature, or, in other words, is absent at all. Therefore, we have used ten-level integrated assessment scale to evaluate the readiness for social and personal orientation of the pupils with disabilities, which is given above. Analysis of the results obtained shows that among tested pupils 3 persons have a high maturity level of all components; 1 pupil has two readiness 
components, corresponding to a high level, 1 pupil has the average-level component (this corresponds to activity level component); 8 pupils have an average maturity level of all components; 20 informants have a low maturity level of all components; whereas 8 pupils show the lack of one of the components at all.

\section{Discussion}

The public attitude towards the children with disabilities in today's Russian society has changed. It is important that today they are incorporated into the contemporary society with due account for their psycho-pedagogical features (Maller, 2006; Sokolov and Kalinikova, 2005). In this context, the adaptive mechanisms, allowing consciously carrying out the social adjustment process, are of special importance (Bgazhnokova and Gamayunova, 2001; Zavrazhin and Fortova, 2005). Such mechanisms include the social and personal orientation, which is understood as a product of the socialization process and socialization result, which provides the opportunity to perceive the social environment, be involved into this environment and to master a variety of activities. Therefore, the formation of readiness for social and personal orientation in lower grades pupils, is of special importance. The readiness structure can be represented by three components (personal, cognitive, and activity component). In order to assess the maturity of these components, we defined criteria and indicators that allowed us to distinguish four levels of maturity (high, medium, low, and immature) of each readiness component. Assessment parameters can be summarized as follows: 1) maturity of readimness components (personal, cognitive and activity), and 2) the integrity of readiness structure.

\section{Conclusion}

Summarizing and systematizing the obtained results, it can be stated that all readiness components for social and personal orientation of the majority of pupils with disabilities are low, and therefore it is impossible to talk about its complete integrity, because high maturity level is reached by just one, at least, two components, constituent its structure.

Contemporary reality has set to the education a task to prepare pupils with disabilities to life in society. November 20, 2014 the regular meeting of the State Duma Committee on Education took place, in which the First Deputy Minister of Education and Science of the Russian Federation N.V. Tretiak said that "we are moving steadily towards ensuring every Russian child with quality and affordable education" (Report of the Government of the Russian Federation of the Federal Assembly of the Russian Federation on the implementation of the state policy in the sphere of education, 2014). A Federal State Educational Standard for primary education (GEF LEO) was created to solve this problem, which provides for the education of children with disabilities in organizations engaged in educational activities adapted to the basic educational programs (AOOP), supplemented by a program of remedial work aimed at developing social (life) student competence and support in the development of the basic educational program primary education (PLO LEO). Organization of educational activities in the PLO LEO can be based on the differentiation of content, taking into account the educational needs and interests of pupils, providing an in-depth study of individual subjects, subject areas PLO LEO (Order "On Amendments to the Order of the Ministry of Education and Science of the Russian Federation dated October 6, 2009. \# 373" "On approval and enactment of the federal state educational standard of primary education", 2014). The most common result of the mastering of the basic educational program by children with disabilities should be an introduction to the culture of the child, for various reasons falling from his educational space that can be implemented using the remedial work on the formation of personality traits such as social and domestic orientation.

\section{Acknowledgements}

This article was prepared under the financial support of the Ministry of Education and Science of the Russian Federation out of the funds of the Project 2.1.2 "Solution to the comprehensive problems of development of professional competence of the teacher and psychologist in continuing education", as well as Strategic Development Program for 2012-2016 of the Mordovian State Pedagogical Institute (MordSPI).

\section{References}

Abbott, L. (2007). Northern Ireland Special Educational Needs Coordinators creating inclusive environments: An epic struggle. European Journal of Special Needs Education, 22(4), 391-407. http://dx.doi.org/10.1080/08856250701650003

Allan, J. (2006). The repetition of exclusion. International Journal of Inclusive Education, 10, 121-13. $\mathrm{http}: / / \mathrm{dx}$.doi.org/10.1080/13603110500221511

Al-Rossan, F. (2012). Introduction to special education. Amman: Dar Al-fker. 
Andreeva, G. M. (2009). Psychology of social cognition. M.: Aspect Press, 303.

Bgazhnokova, I. M. (2001). Social adjustment of orphans with intellectual disabilities. Psychological and pedagogical problems of social adjustment of children with intellectual disabilities, M.: MIPKRO, 4-21.

Humphrey, N. (2008). Including pupils with autistic spectrum disorders in mainstream schools. Support for Learning, 23(1), 41-7. http://dx.doi.org/10.1111/j.1467-9604.2007.00367.x

Lynch, S., \& Irvine, A. (2009). Inclusive education and best practice for children with autism spectrum disorder: An integrated approach. International Journal of Inclusive Education, 13(8), 845-89. http://dx.doi.org/10.1080/13603110802475518

Makarova, L. N. (2000). High school teacher: personality, style, activity. Part 1. Tambov: Publishing House of Tambov State University, 243.

Maller, A. R. (2006). Child with Disabilities: A book for parents. M.: Pedagogy Press, 284.

Malofeev, N. N. (2010). Special education in a changing world. M.: Education, 319.

Mintz, J. (2007). Attitudes of primary initial teacher training students to special educational needs and inclusion. Support for Learning, 22(1), 3-8. http://dx.doi.org/10.1111/j.1467-9604.2007.00438.x

Mitchell, D. (2004). Special educational needs and inclusive education. London: Routledge.

Nazarova, N. M. (2000). Special Pedagogy. Moscow: Academy, 519.

Norwich, B. (2008). Dilemmas of difference, inclusion and disability. Oxon: Routledge.

Parfyonova, T. A. (2013). Technology of social and personal orientation of pupils with disabilities in terms of integrated education. Mordovian State Pedagogical Institute: Saransk, 124.

Rogers, C. (2013). Inclusive education and intellectual disability: a sociological engagement with Martha Nussbaum. International Journal of Inclusive Education, 17(9), 988-002. http://dx.doi.org/10.1080/13603116.2012.727476

Ryabova, N. V. (2007), Pedagogical grounds of social and personal orientation of the mentally challenged pupil. Saransk, 240

Ryabova, N. V. (2011). Technologies of formation of social and personal orientation of a child with disabilities. Siberian Pedagogical Journal, 8, 82-89.

Shipitsyna, L. M. (2003). Psychological and pedagogical advice and support of child development. M.: VLADOS, 528.

Shmatko, N. D. (2010). Joint education and training of children with disabilities and normally developing preschool children. Care and education of children with disabilities, 5, 12-19.

Sokolova, N. D. (2005). Children with disabilities: problems and innovative trends in training and education. M.: Gnom \& D, 180.

Thomazet, S. (2009). From integration to inclusive education: does changing the terms improve practice? International Journal of Inclusive Education, 13(6), 553-563. http://dx.doi.org/10.1080/13603110801923476

Zavrazhin, S. A. (2005). Adjustment of children with disabilities. M.: Triksta, 400.

\section{Copyrights}

Copyright for this article is retained by the author(s), with first publication rights granted to the journal.

This is an open-access article distributed under the terms and conditions of the Creative Commons Attribution license (http://creativecommons.org/licenses/by/3.0/). 